\title{
Electron acceleration by a radially polarized laser pulse during ionization of low density gases
}

\author{
Kunwar Pal Singh ${ }^{1,2, *}$ and Manoj Kumar ${ }^{2}$ \\ ${ }^{1}$ School of Physics, University of Sydney, New South Wales 2006, Australia \\ ${ }^{2}$ Singh Simutech Pvt. Ltd., Bharatpur, Rajasthan, 321201, India \\ (Received 25 September 2010; published 7 March 2011)
}

\begin{abstract}
The acceleration of electrons by a radially polarized intense laser pulse has been studied. The axial electric field of the laser is responsible for electron acceleration. The axial electric field increases with decreasing laser spot size; however, the laser pulse gets defocused sooner for smaller values and the electrons do not experience high electric field for long, reducing the energy they can reach. The electron remains confined in the electric field of the laser for longer and the electron energy peaks for the normalized laser spot size nearly equal to the normalized laser intensity parameter. Electron energy peaks for initial laser phase $\phi_{0}=\pi$ due to accelerating laser phase and decreases with transverse initial position of the electrons. The energy and angle of the emittance spectrum of the electrons generated during ionization of krypton and argon at low densities have been obtained and a right choice of laser parameters has been suggested to obtain high energy quasimonoenergetic collimated electron beams. It has been found that argon is more suitable than krypton to obtain high energy electron beams due to higher ionization potential of inner shells for the former.
\end{abstract}

DOI: 10.1103/PhysRevSTAB.14.030401

PACS numbers: 52.59.Bi, 42.65.- $\mathrm{k}, 52.75 . \mathrm{Di}$

\section{INTRODUCTION}

Beams of charged particles are used in different applications ranging from tabletop narrow band femtosecond $\mathrm{x}$-ray sources, free-electron lasers, subatomic matter interactions [1], material science, nuclear reactions, cancer treatment, and medicine. Laser driven particle acceleration has been proposed as a compact and low-cost alternative to the conventional acceleration schemes [2-4]. Intense short-pulse lasers have been developed with light intensities as high as $10^{22} \mathrm{~W} / \mathrm{cm}^{2}$ with the development of chirp-pulse-amplification technique. A number of methods, such as the cyclotron autoresonance [5], inverse freeelectron laser [6], the inverse Cherenkov accelerator [7], the laser wake-field accelerator [8], the plasma beat wave accelerator [9], the vacuum beat wave accelerator [10], and electron acceleration by laser field in vacuum [11], have been proposed as laser based acceleration schemes. Direct electron acceleration in vacuum has been investigated experimentally [12] and theoretically [13].

Linearly polarized laser beams have predominantly been used in previous investigations on laser driven electron acceleration in vacuum. When the quiver amplitude of the electrons driven by the laser field exceeds the focal spot radius of a Gaussian beam, the restoring force acting on the electron decays exponentially, and the electron

\section{*k_psingh@yahoo.com}

Published by American Physical Society under the terms of the Creative Commons Attribution 3.0 License. Further distribution of this work must maintain attribution to the author(s) and the published article's title, journal citation, and DOI. escapes with high energy. The electrons close to the temporal peak of the laser pulse show strong initial phase dependence for a linearly polarized laser pulse [14]. Energy enhancement by a circularly polarized laser pulse during acceleration of the electrons by a Gaussian laser pulse was demonstrated by Singh et al. [15]. Energy enhancement was attributed to the axial symmetry of the circularly polarized pulse. The electron acceleration by radially polarized laser has attracted attention in recent years due to its capability to produce electron beams with high energy, narrow divergence, and narrow energy spreads. The electrons gain net energy in vacuum if a radially polarized laser is focused to a small spot size. Focusing of the laser causes all the components of electric and magnetic field to appear in the focal region [16-18]. For a tightly focused radially polarized laser pulse, the strong electric field component in the direction of propagation (longitudinal component) of the laser plays an important role in accelerating the electron [19-23]. The electrons at the beam axis are accelerated to relativistic energies along the laser axis by the longitudinal electric field. The lowest-order radially polarized fields of a Gaussian laser beam were employed and capability of radially polarized laser beam to accelerate electrons to $\mathrm{GeV}$ energies in vacuum was shown [24]. A relativistic single particle simulation of vacuum acceleration of an electron by a high-intensity radially polarized laser beam was presented [25] and it was shown that an external magnetic field further enhances the energy of the accelerated electrons. Three-dimensional particle-in-cell (3D-PIC) simulations of electron acceleration in vacuum with radially polarized ultraintense laser beams were 
performed by Karmakar and Pukhov [26] and it was shown that high quality electron beams with narrow divergence and narrow energy spreads can be produced using radially polarized laser pulse. It was found that the electrons generated close to the rising edge of the laser pulse (for low- $Z$ materials) never experience the peak of the pulse and gain low energies. A single-cycle laser pulse efficiently accelerates electrons to $\mathrm{GeV}$ energies. One has to employ ionization of high- $Z$ materials to inject electrons in the accelerating phase at the laser pulse maximum for long laser pulses.

A radially polarized laser can be produced using different methods. The generation of a radially polarized beam by inserting an undoped $c$-cut $\mathrm{YVO}_{4}$ crystal into a $\mathrm{Nd}$ : YAG laser cavity was demonstrated [27]. A Brewster optical element consisting of convex and concave conical prisms was designed and fabricated to generate a radially polarized laser beam [28]. A high power radially polarized beam was produced directly from a diode-pumped $\mathrm{Nd}: \mathrm{YVO}_{4}$ bounce amplifier, using an autocloned photonic crystal mirror as an output coupler, using a simple cavity configuration [29]. Experimental results were presented on the intracavity generation of radially polarized light by incorporation of a polarization-selective mirror in a $\mathrm{CO}_{2}$-laser resonator [30].

Recently, electron acceleration by a radially polarized laser pulse was studied by Fortin et al. [31]. They found the dependence of electron energy on laser power, beam waist size, and the pulse duration for optimum acceleration. They have confined their study for the electrons placed on the laser propagation axis for which the longitudinal field dominates acceleration. However, during the experimental process on axis as well as off axis electrons are involved and contribute to the beam quality. Therefore, it is important to consider on axis as well as off axis electrons.

It is important to understand parametric dependence of energy to overcome the limitations related to beam quality and enhance the energy. We find dependence on different parameters of electron acceleration by a radially polarized intense Gaussian laser pulse considering on axis as well as off axis electrons which has not been done in detail in previous studies. We investigate electron acceleration during ionization of low density gases (which is intermediate regime between plasma and vacuum) by a radially polarized laser pulse which has not been studied until now to the best of our knowledge. The energy and angle of emittance spectrum of the electrons generated during ionization of krypton and argon at low densities has been obtained and a right choice of laser parameters has been suggested to obtain high energy quasimonoenergetic collimated electron beams. This paper is organized as follows. The next section describes the electromagnetic fields and electron dynamics used to study electron acceleration. Results and discussion are described in Sec. III. Finally, conclusions are drawn in the last section.

\section{ELECTROMAGNETIC FIELDS AND ELECTRON DYNAMICS}

The laser pulse is assumed radially polarized propagating along the $z$ axis with following electric field:

$$
\mathbf{E}=\hat{r} E_{r}+\hat{z} E_{z},
$$

where $E_{r}=E_{0} \frac{r}{r_{0} f^{2}} \exp \left(-\left\{\left[t-\left(z-z_{L}\right) / c\right]^{2} / \tau^{2}\right\}-\left[r^{2} /\right.\right.$ $\left.\left.\left(r_{0}^{2} f^{2}\right)\right]\right) \cos (\phi)$, and $E_{z}=E_{0} \frac{2}{r_{0} f^{2}} \exp \left(-\left\{\left[t-\left(z-z_{L}\right) / c\right]^{2} /\right.\right.$ $\left.\left.\tau^{2}\right\}-\left[r^{2} /\left(r_{0}^{2} f^{2}\right)\right]\right)\left(\left\{1-\left[r^{2} /\left(r_{0}^{2} f^{2}\right)\right]\right\} \sin (\phi)-\frac{z}{Z_{R}}\left[r^{2} /\left(r_{0}^{2} f^{2}\right)\right] \times\right.$ $\cos (\phi))$, where $\phi=\omega t-k z+2 \tan ^{-1}\left(z / Z_{R}\right)-\frac{z}{Z_{R}} \times$ $\left[r^{2} /\left(r_{0}^{2} f^{2}\right)\right]+\phi_{0}, f^{2}=1+\left(z / Z_{R}\right)^{2}, Z_{R}=k r_{0}^{2} / 2$ is the Rayleigh length, $\tau$ is pulse duration, $r_{0}$ is spot size of the laser, $r$ is transverse (radial) coordinate, and $z_{L}$ is the initial position of the pulse peak.

The magnetic field related to the laser pulse is given by Maxwell's equation $\nabla \times \mathbf{E}=-\partial \mathbf{B} / \partial t$ :

$$
B_{r}=0, \quad B_{\theta}=\frac{E_{r}}{c}, \quad B_{z}=0 .
$$

The initial positions of the electrons are assumed at $z_{i}, r_{i}$ and a radially polarized laser pulse with initial position of the peak at $z_{L}$ propagates towards the electrons and accelerates them to high energy. The initial position of the electrons is assumed ahead of the laser pulse peak in the $z$ direction so that as the laser pulse propagates, it interacts with them.

The equations governing electron momentum are obtained from relativistic Newton-Lorentz equation of motion $d \mathbf{P} / d t=-e(\mathbf{E}+\mathbf{v} \times \mathbf{B})$,

$$
\begin{aligned}
& d p_{r} / d t=-e\left(E_{r}-v_{z} B_{\theta}\right), \\
& d p_{z} / d t=-e\left(E_{z}+v_{r} B_{\theta}\right) .
\end{aligned}
$$

The equation governing electron energy is given by

$$
m_{0} c^{2} \frac{d \gamma}{d t}=-e\left(v_{r} E_{r}+v_{z} E_{z}\right)
$$

where $-e$ and $m_{0}$ are electron charge and rest mass, respectively.

The normalized laser intensity parameter is given by $a_{0}=e E_{0} / m_{0} \omega c, a_{r}=e E_{r} / m_{0} \omega c, a_{z}=e E_{z} / m_{0} \omega c$, and normalized magnetic field by $b_{r, \theta, z}=e B_{r, \theta, z} / m_{0} \omega$. Throughout this paper time, length, and velocity are normalized by $1 / \omega, 1 / k$, and $c$, respectively.

Equations (3)-(5) are simplified using $p_{r}=\gamma m_{0} v_{r}$, $p_{z}=\gamma m_{0} v_{z}, \gamma^{2}=1+\left(p_{r}^{2}+p_{z}^{2}\right) / m_{0}^{2} c^{2}$. We obtain the following equations:

$$
\begin{aligned}
& \gamma \frac{d v_{r}}{d t}=\left(v_{r}^{2}-1\right) a_{r}+v_{z} b_{\theta}+v_{r} v_{z} a_{z}, \\
& \gamma \frac{d v_{z}}{d t}=\left(v_{z}^{2}-1\right) a_{z}-v_{r} b_{\theta}+v_{z} v_{r} a_{r} .
\end{aligned}
$$

Equations (6) and (7) are coupled ordinary differential equations, which have been solved numerically by a 
two-dimensional acceleration simulation code to obtain electron trajectory and momentum. The code uses the fifth order Runge-Kutta method with an adaptive time step size control to achieve desired accuracy of the solution. The implementation of the modified algorithm for the RungeKutta method results in nearly 3 times reduction in computational time over the classical Runge-Kutta method with an adaptive time step size control.

The longitudinal component $E_{z}$ is small compared to radial component $E_{r}$ for wide laser spot size; however, its value becomes significant for tightly focused pulses and plays a very important role during electron acceleration. In fact, it is responsible for acceleration of electrons for a radially polarized laser pulse. Figures 1 (a) and 1(b) show radial field intensity as a function of normalized $r$ for normalized laser spot sizes $r_{0}=5,10,15$, and 20 and spatial distribution of radial field intensity, respectively. The radial field intensity increases with $r$ and peaks at $z=0$ and $r= \pm r_{0} / \sqrt{2}$. The spatial distribution of radial
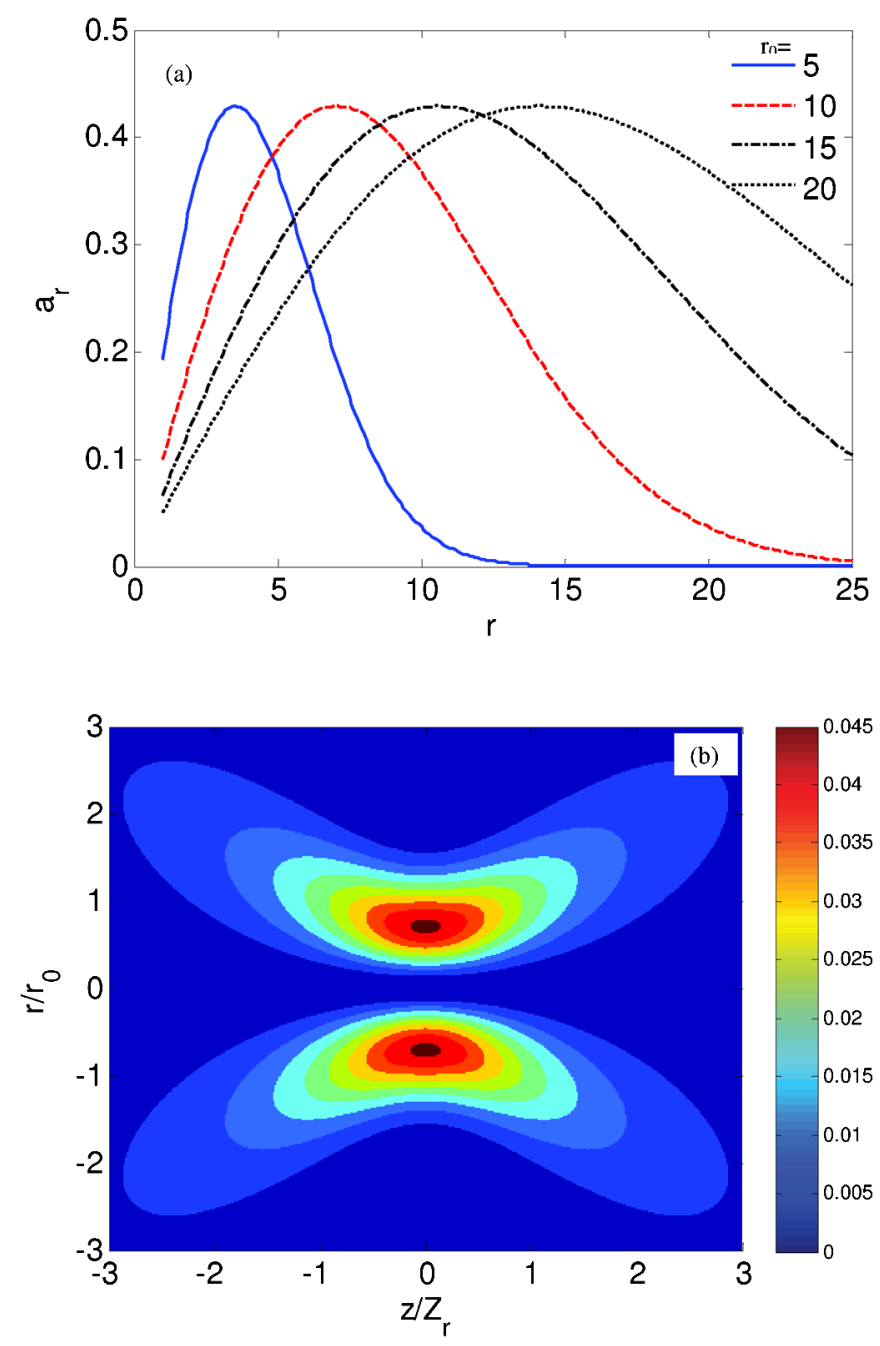

FIG. 1. (a) Normalized radial field intensity as a function of $r$ at $z=0$. (b) The spatial distribution of radial field intensity for $r_{0}=10$. Normalized laser intensity parameter $a_{0}=1$.
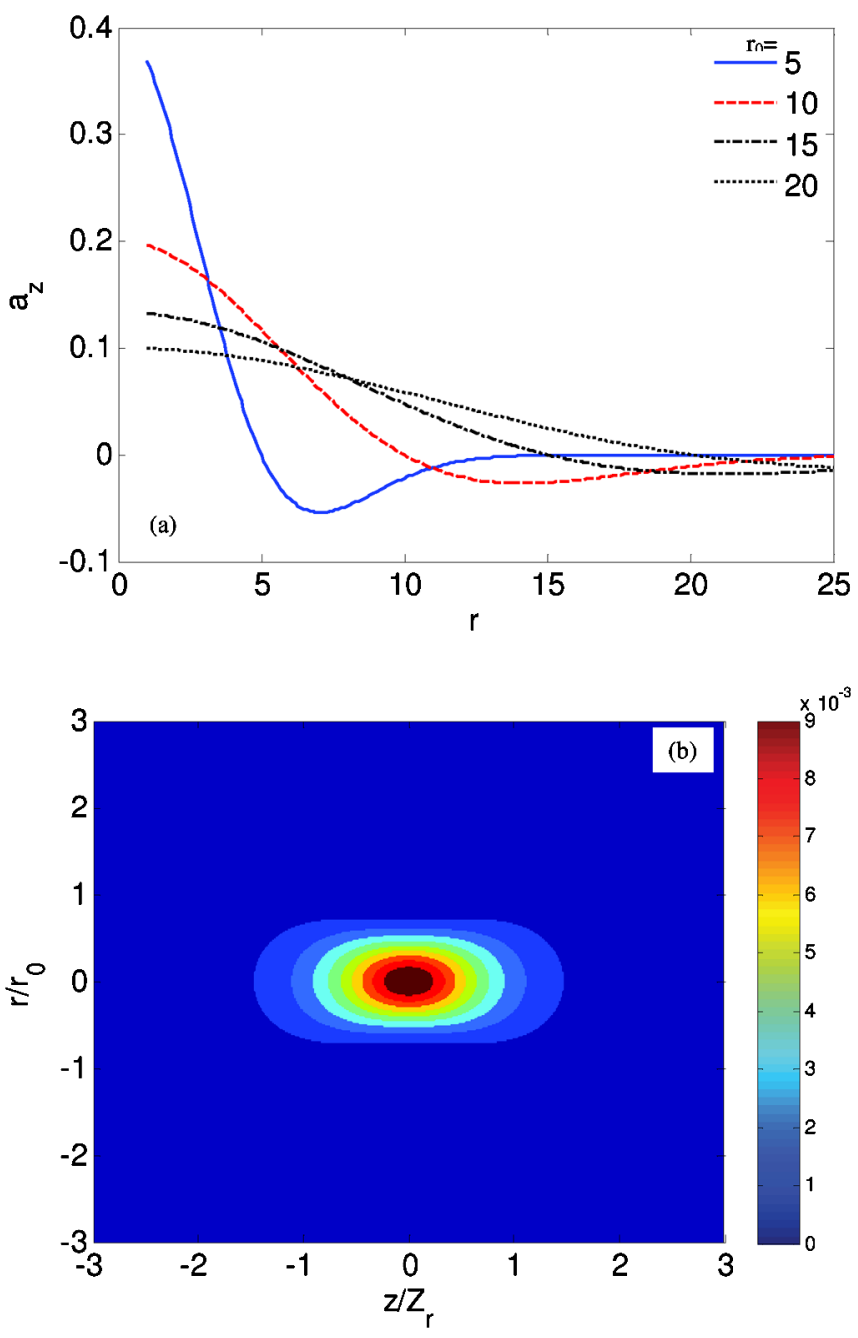

FIG. 2. (a) Normalized axial field intensity as a function of $r$ at $z=0$. (b) The spatial profile of the axial field intensity for $r_{0}=10$. Normalized laser intensity parameter $a_{0}=1$.

field intensity shows two lobes like structures. Figures 2(a) and 2(b) show the axial field intensity as a function of normalized $r$ for normalized laser spot size $r_{0}=5,10,15$, and 20 and spatial distribution of axial field intensity, respectively. The axial field intensity is highest at the origin $(z=0, r=0)$.

\section{RESULTS AND DISCUSSION}

This section is divided in two subsections: Sec. III A describes electron acceleration in vacuum and Sec. III B describes acceleration of electrons generated during ionization of krypton and argon.

\section{A. Electron acceleration in vacuum}

In this subsection, we describe variation of electron energy with laser parameters and initial conditions of an electron. We have restricted our study to the electrons with zero initial velocity because the electrons are generated 
with this velocity during ionization of atoms. The results are reported at normalized final time at $t_{f}=10^{5}$. Default parameters are normalized pulse duration $\tau=200$, initial position of pulse peak $z_{L}=0$, initial electron position $z_{0}=0$ and $r_{i}=0$, unless stated otherwise. Dotted, dashed, dot-dashed, and solid lines are for $a_{0}=25,50,75$, and 100, respectively, for Figs. 3-5.

Figures 3(a) and 3(b) show electron trajectory at $r_{i}=$ $r_{0} / 2$ for $r_{0}=a_{0} / 2$ and $r_{0}=a_{0}$, respectively. The electrons follow a nearly straight line path indicating that acceleration is mainly due to the longitudinal electric field of the radially polarized laser pulse. The scattering of the electron reduces with an increase in the laser spot size. Figures 4(a) and 4(b) show electron energy $K(\mathrm{GeV})$ as a function of normalized $z$ at $r_{i}=r_{0} / 2$ for $r_{0}=a_{0} / 2$ and $r_{0}=a_{0}$, respectively. The energy decreases for the electrons originating away from the axis. This is because the axial field intensity of a radially polarized laser pulse decreases with an increase in the value of $r$ at $z=0$ as

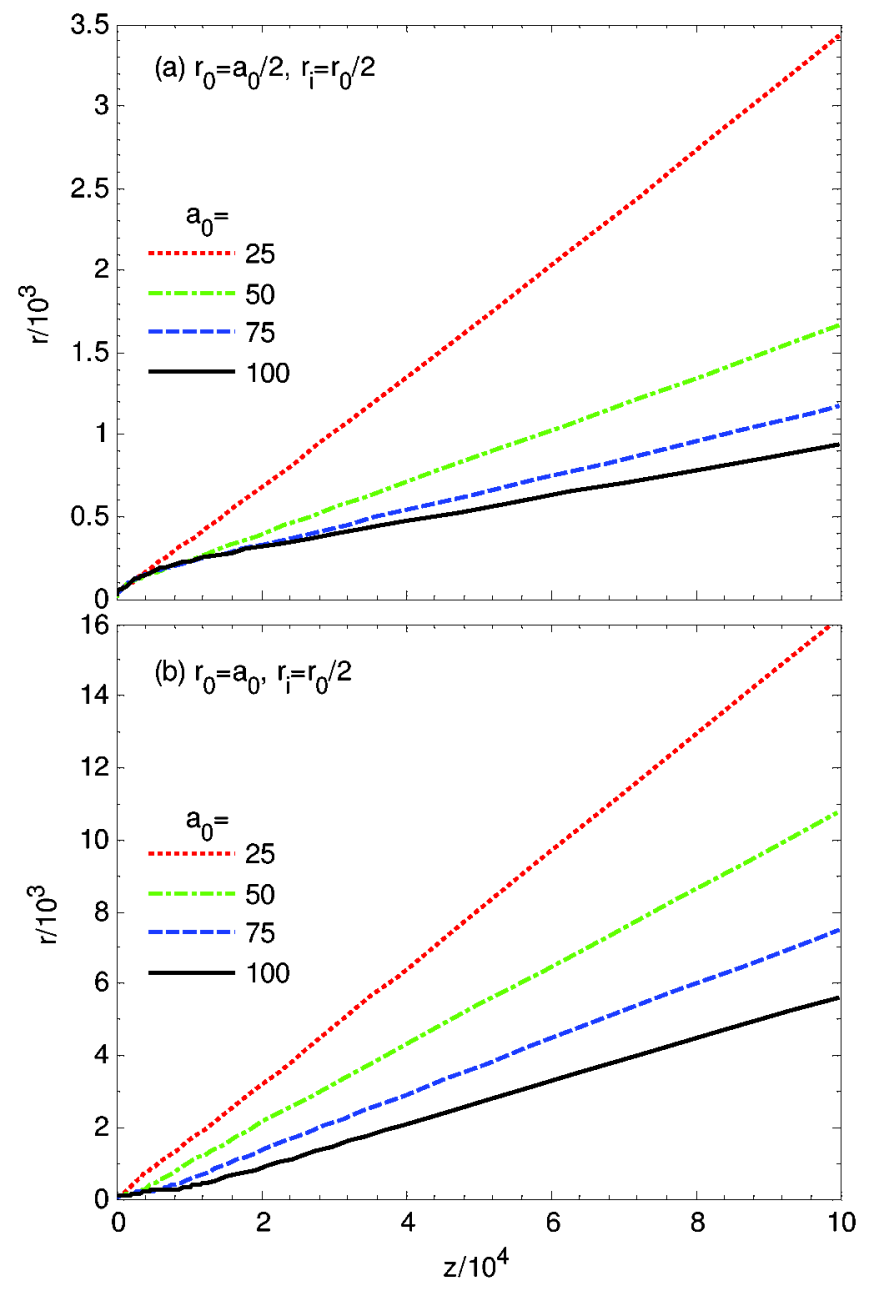

FIG. 3. Electron trajectory for normalized $r$ and $z$ for (a) $r_{0}=a_{0} / 2$ and (b) $r_{0}=a_{0}$ for $r_{i}=r_{0} / 2$. Dotted, dashed, dot-dashed, and solid lines are for $a_{0}=25,50,75$, and 100, respectively. The parameters are $\tau=200, z_{L}=0$, and $t_{0}=0$.
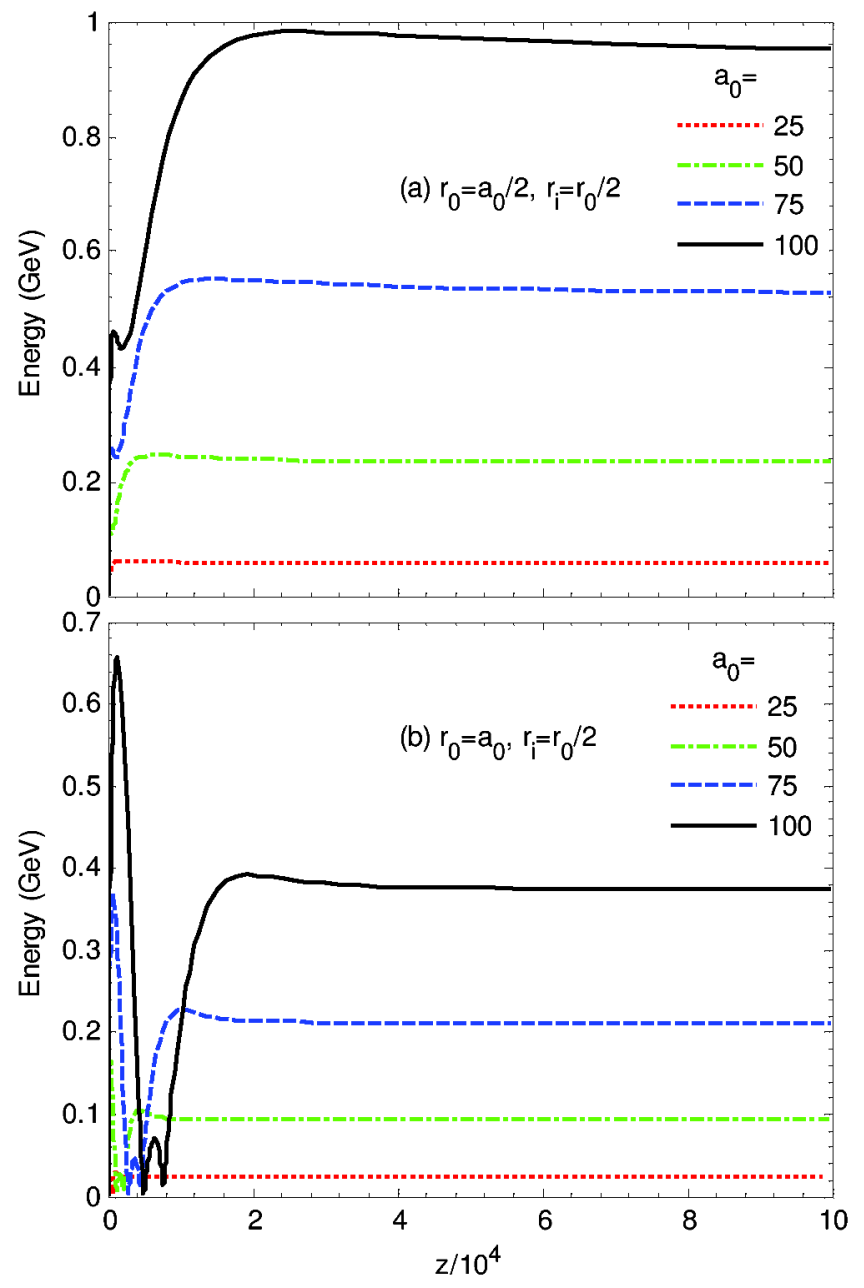

FIG. 4. Electron energy $K(\mathrm{GeV})$ as a function of normalized $z$ for (a) $r_{0}=a_{0} / 2$ and (b) $r_{0}=a_{0}$ for $r_{i}=r_{0} / 2$. Dotted, dashed, dot-dashed, and solid lines are for $a_{0}=25,50,75$, and 100, respectively. The parameters are $\tau=200, z_{L}=0$, and $t_{0}=0$.

can be seen from Fig. 2. The electron gains nearly $0.9 \mathrm{GeV}$ energy for $r_{0}=a_{0} / 2$ and nearly $0.38 \mathrm{GeV}$ for $r_{0}=a_{0}$. We observe kinks in the energy curve which can be attributed to the complex trajectory of electron with $r_{i}=r_{0} / 2$ under the influence of the laser field. When the electron is closer to axis, energy is higher and vice versa. The phase dependence of the energy may also be playing some role in this process. Figures 5(a) and 5(b) show electron energy $K$ $(\mathrm{GeV})$ as a function of $z$ at $r_{i}=0$ for $r_{0}=a_{0} / 2$ and $r_{0}=a_{0}$, respectively. The energy of the electron is higher for $r_{0}=a_{0}$. The electron gains nearly $1.9 \mathrm{GeV}$ energy for $r_{0}=50$ and nearly $2.7 \mathrm{GeV}$ for $r_{0}=100$ for $a_{0}=100$. The electron remains trapped for longer for laser spot size $r_{0}=a_{0}$ than that for $r_{0}=a_{0} / 2$. This is because for a higher value of laser spot size, the laser defocuses slowly and the electron remains confined in the laser fields for longer, reducing electron scattering and enhancing energy gain. 


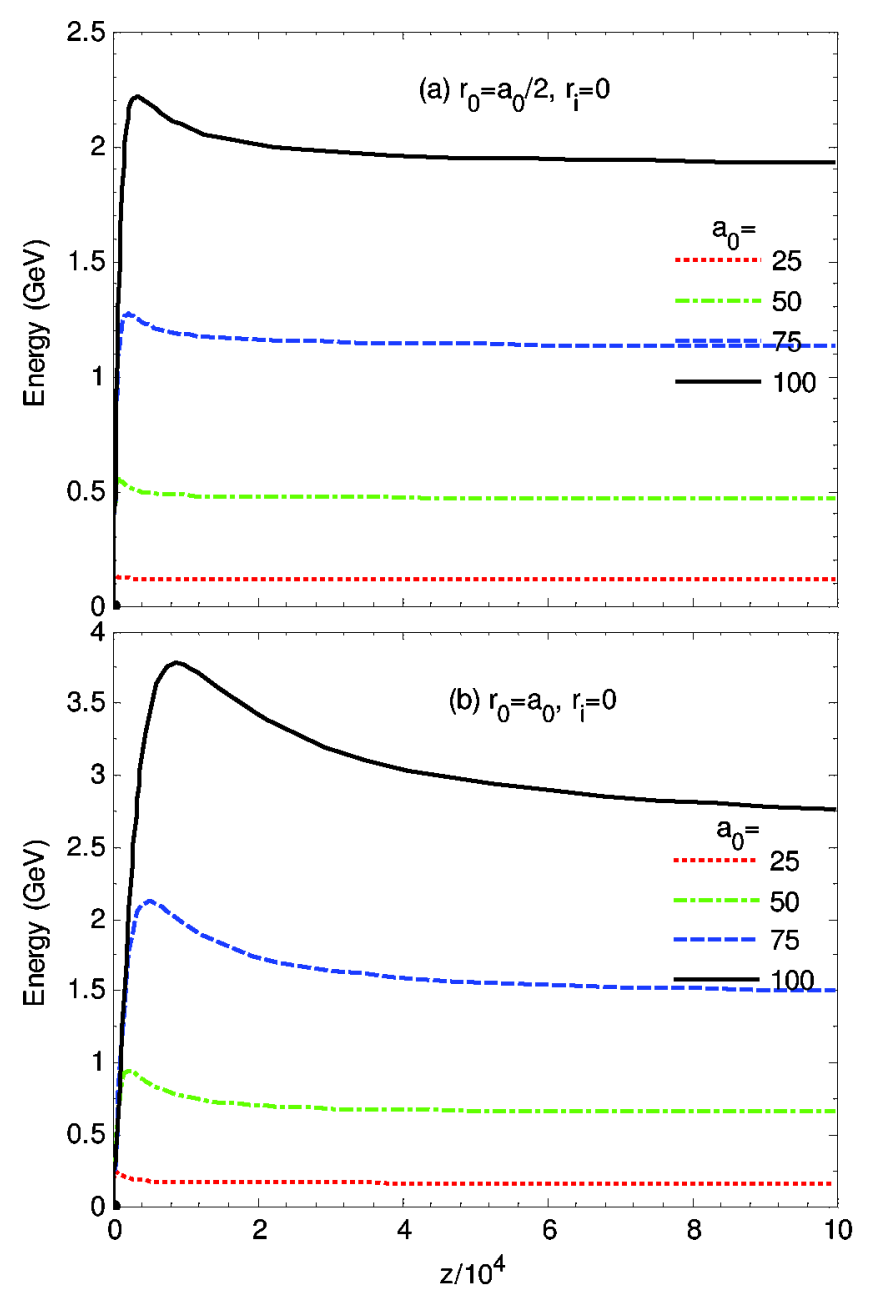

FIG. 5. Electron energy $K(\mathrm{GeV})$ as a function of normalized $z$ for (a) $r_{0}=a_{0} / 2$ and (b) $r_{0}=a_{0}$ for $r_{i}=0$. Dotted, dashed, dot-dashed, and solid lines are for $a_{0}=25,50,75$, and 100, respectively. The parameters are $\tau=200, z_{L}=0$, and $t_{0}=0$.

Figures 6(a) and 6(b) show electron energy distribution as a function of normalized $r_{0}$ and initial phase $\phi_{0}$ for $a_{0}=25$, and $a_{0}=75$, respectively. The electron energy peaks for $\phi_{0}=\pi$ and $r_{0}=25$ for $a_{0}=25$ and $r_{0}=75$ for $a_{0}=75$. The highest electron energy is nearly $140 \mathrm{MeV}$ and $1.4 \mathrm{GeV}$ for $a_{0}=25$ and $a_{0}=75$, respectively. The optimum value of laser spot size is nearly equal to the laser intensity parameter. When $r_{0}>a_{0}$, longitudinal electric field is weak and when $r_{0}<a_{0}$ laser pulse diverges fast reducing interaction duration; therefore, the energy peaks for $r_{0}=a_{0}$. The longitudinal component of the electric field of the laser $E_{z}$ is responsible for accelerations. It can be seen from Eq. (1) that $E_{z}$ is proportional to $\sin (\phi)$ for $z=0$, which becomes negative for initial phase $\phi_{0}>\pi$ and electric force on electrons becomes positive in the $z$ direction. Hence, the initial phase $\phi_{0}=\pi$ is the accelerating phase and an electron gains highest energy for it. There are two more points $\phi_{0}=$ $0.67 \pi$ and $\phi_{0}=1.34 \pi$ around which electron energy is
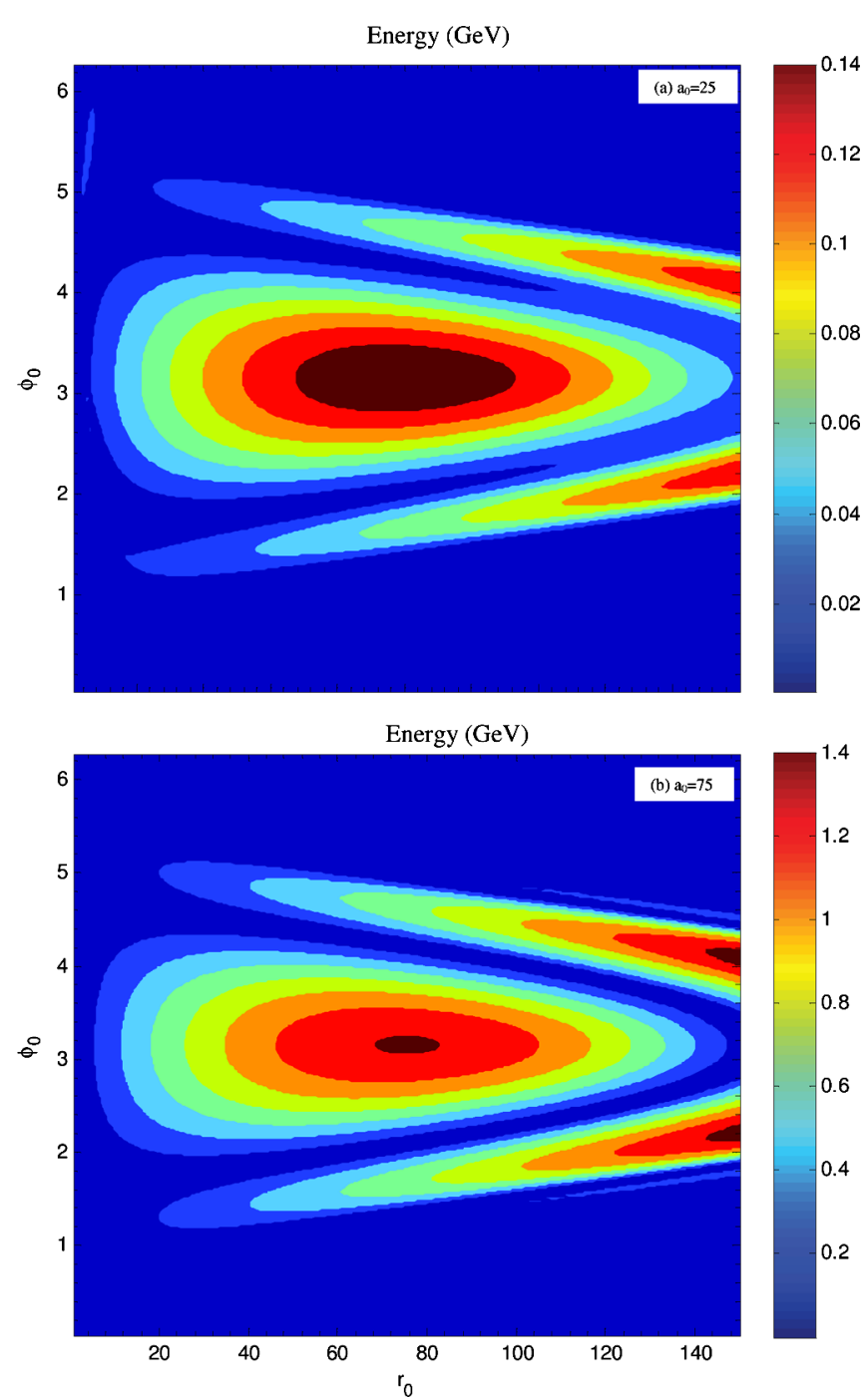

FIG. 6. Electron energy (in $\mathrm{GeV}$ ) distribution as a function of normalized $r_{0}$ and $\phi_{0}$ for (a) $a_{0}=25$, and (b) $a_{0}=75$. Other parameters are $r_{i}=0, t_{0}=0, \tau=200, z_{L}=0$, and $z_{0}=0$.

close to peak energy. The value of laser spot size $r_{0}$ is too high for these points and will require very high power laser, therefore, not of interest.

Normalized ionization time $t_{0}$ and the position of normalized laser pulse peak $z_{L}$ are closely associated with each other and affect energy gain. We have considered these two parameters to know their effect on energy gain in Fig. 7. Figures 7(a) and 7(b) show electron energy distribution as a function of normalized ionization time $t_{0}$ and initial position of the pulse peak $z_{L}$ for $a_{0}=25$ and $a_{0}=75$, respectively. We can see bands of high energy parallel to the $z_{L}$ axis. The electron gains highest energy when the initial position of the pulse peak is nearly equal to the normalized ionization time $t_{0}$. The gas atoms interact with the rising part of the laser pulse in the beginning (after $t=0$ ) and then the peak of the laser pulse approaches them as the interaction time progresses. The trailing part of the 

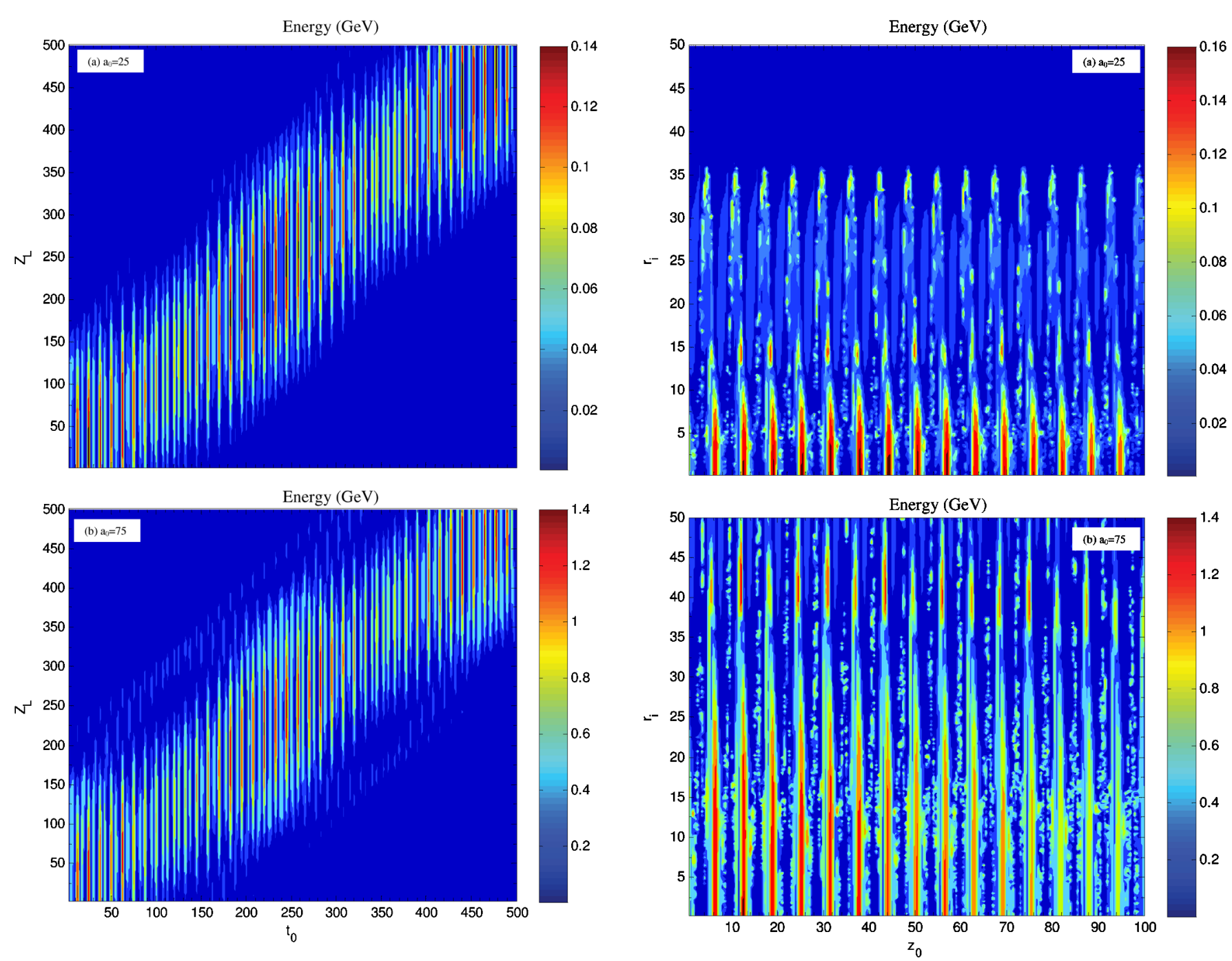

FIG. 7. Electron energy (in $\mathrm{GeV}$ ) distribution as a function of normalized $t_{0}$ and $z_{L}$ for (a) $a_{0}=25$, and (b) $a_{0}=75$. Other parameters are $r_{i}=0, r_{0}=a_{0}, \tau=200, \varphi_{0}=\pi$, and $z_{0}=0$.

laser pulse interacts with gas atoms last. The electrons are generated only up to the peak of the pulse. The electrons with low ionization time are the electrons generated by the rising edge of the pulse and the electrons with higher values of ionization time are the electrons generated close to the peak of the pulse. Thus, maximum possible ionization occurs when the peak of the laser pulse interacts with atoms. The interaction of the gas atoms with the trailing part does not contribute to the ionization and generation of the electrons, due to its lower intensity than the peak of the pulse. If we use a high atomic number gas, the electrons are generated throughout the interaction from the rising edge to the peak of the laser pulse. The electrons generated away from laser pulse peak do not gain significant energy. The electrons are generated during interaction of high-intensity laser pulse with gas atoms through multiple ionizations.

Figures 8(a) and 8(b) show electron energy distribution as a function of normalized initial position of the electron

FIG. 8. Electron energy (in $\mathrm{GeV}$ ) distribution as a function of normalized $z_{0}$ and $r_{i}$ for (a) $a_{0}=25$, and (b) $a_{0}=75$. Other parameters are $t_{0}=0, z_{L}=0, r_{0}=a_{0}, \tau=200$, and $\varphi_{0}=\pi$.

$z_{0}$ and $r_{i}$ for $a_{0}=25$ and $a_{0}=75$, respectively. It can be seen that electron energy decreases with an increase in the value of $r_{i}$. The electron energy is highest for some specific values of $z_{0}$. This is because the initial phase of the laser with respect to electron depends on the value of initial electron position $z_{0}$ and electron energy depends on initial phase. Therefore, we see small bands in the energy as a result of the initial phase dependence of the energy. Energy of the electrons decreases with an increase in the value of $z_{0}$ because these electrons are away from the peak of the laser pulse.

\section{B. Acceleration of electrons generated during ionization of krypton and argon}

This subsection describes acceleration of electrons generated during ionization of krypton and argon gas atoms. Short-pulse, high field ionization at infrared frequencies 
typically occurs in the quasiclassical regime in which the field distorts the Coulomb binding potential allowing tunneling of electrons from a bound to a free state. One of the accurate tunneling models in this regime is the AmmosovDelone-Krainov tunneling model [32]. At very high fields, tunneling can be approximated by its classical limit where electrons become free from the Coulomb barrier to a free state. This model is known as barrier suppression ionization and predicts ionization threshold intensities of tightly bound electrons quite well. We define normalized ionization time $t_{0}$ as the instance at which an electron is generated. The ionization time depends upon laser intensity, atomic number of the atom, and ionization potential of the electrons and can be obtained using the following relation:

$$
I_{\mathrm{th}}\left(\mathrm{W} / \mathrm{cm}^{2}\right)=4 \times 10^{9} \frac{I_{p}^{4}(\mathrm{eV})}{Z^{2}},
$$

where $I_{p}$ is the ionization potential, $Z$ is the atomic number, and $I_{\text {th }}$ is the threshold laser intensity. Above-threshold ionization is primarily the result of the interaction of a newly freed electron with the laser field in the longwavelength limits and classical treatment of physics predicts that linearly and circularly polarized light generate different above-threshold ionization spectra [33]. The theoretical description and the experimental methods and results for above-threshold ionization by few-cycle pulses have been reviewed [34]. Ivanov et al. [35] discussed the mechanisms of multielectron dissociative ionization of diatomic molecules in intense laser fields and showed that during dissociation of molecular ions the nuclei pass through a critical range of internuclear distances where ionization is enhanced by several orders of magnitude for several successive charge states of the molecule.

As time progresses, the peak of the pulse approaches the atoms and more electrons are produced through multiple ionization. It has been found experimentally that plasma effects can be neglected at pressures below 1 torr [36]. At this pressure, plasma density is nearly $10^{23} / \mathrm{m}^{3}$. The plasma frequency is $\omega_{\text {pe }}=5.64 \times 10^{4} \sqrt{10^{17}} \cong$ $1.78 \times 10^{13}$ radian/second. The laser frequency is given by $\omega=2 \pi c / \lambda=1.88 \times 10^{15}$ radian per second for $\lambda=$ $1 \mu \mathrm{m}$, hence, $\omega_{\mathrm{pe}}^{2} / \omega^{2} \cong 10^{-4}$. Plasma effects such as wakefields, plasma instabilities, modification of the laser envelope, etc. can be neglected for this plasma density. The numerical simulations presented in this work are valid in this regime. We have used high laser intensities and total laser energies. The choice is exploratory and the lasers available today do not reach them. Future laser projects like extreme light infrastructure (ELI) will reach them [37]. The following description gives some idea of the relationship between normalized and real laser parameters. Considering normalized laser spot sizes $r_{0}=a_{0}$, real laser spot sizes are nearly $4 \lambda$ and $11.94 \lambda$, corresponding to $a_{0}=$ 25 and 75, respectively. Real pulse durations in femtosec- onds (fs) is nearly $106 \lambda[\mu \mathrm{m}]$ fs corresponding to normalized pulse duration $\tau=200$. Laser intensity is associated with parameter $a_{0}$ by following relation $I\left[\mathrm{~W} / \mathrm{cm}^{2}\right]=$ $1.38 \times 10^{18}\left(a_{0} / \lambda[\mu \mathrm{m}]\right)^{2}$. The laser intensities are nearly $I=8.63 \times 10^{20} \mathrm{~W} / \mathrm{cm}^{2}$ and $7.76 \times 10^{21} \mathrm{~W} / \mathrm{cm}^{2}$, for $a_{0}=25$ and 75, respectively, for $\lambda=1 \mu \mathrm{m}$. Laser power is given by $P=0.54\left(a_{0} r_{0}\right)^{2} \mathrm{GW}$. Laser power is nearly 0.211 petawatt for $a_{0}=25$ and 17.1 petawatt for $a_{0}=75$.

It is well known that the electrons created from the ionization of neutral atoms near the rising edge of the pulse do not gain sufficient energy. Recently, Singh et al. [38] proposed a scheme for the acceleration of electrons generated during the ionization of a gas by two laser pulses. If a prepulse is used before the main pulse then the prepulse removes electrons from the outer shells, and the main laser pulse interacts with the electrons in the inner shells of high atomic number gases, such as krypton and argon. The electrons generated close to the peak of the main laser pulse gain energy in the $\mathrm{GeV}$ with a small spread in the energy and low angle of emittance for a circularly polarized laser pulse. For most applications, high quality electron beams are necessary. Full momentum images of electrons rescattered from $\mathrm{Xe}, \mathrm{Kr}$, and Ar following the liberation of the electrons from these atoms by short intense laser pulses were measured [39].

The energy and angle of emittance spectrum of the electrons generated during ionization of krypton and argon at low densities have been obtained to find out a right choice of laser parameters to obtain high energy quasimonoenergetic collimated electron beams. The initial position of the laser pulse peak is taken at $z_{L}=-300$ and the last normalized time is taken $10^{6}$ for the Figs. 9 and 10. We have taken $5 \times 10^{4}$ atoms of krypton and $10^{5}$ atoms of argon randomly distributed from $r=-r_{0}$ to $+r_{0}$ and $z_{0}=0$ to 100 for the results of Figs. 9 and 10. This number was found sufficient to obtain statistically unchanged energy and scattering spectrum. The $N_{\gamma}$ and $N_{\alpha}$ are obtained by dividing the whole range of energy and angle of emittance in 50 parts and then counting the number of electrons (relative scale) with energy lying within a range of relativistic factor and scattering lying within a range of angle of emittance, respectively, for Figs. 9 and 10 in the following description.

There is significant difference between the ionization energies of 26th $(1205.3 \mathrm{eV})$ and 27th $(2928 \mathrm{eV})$ electrons of the krypton. We can use this difference to obtain high energy electrons by removing 26 electrons in the outer shells of the krypton by a prepulse and then accelerating 27th to 30th electrons of krypton atoms by a main laser pulse as was shown in Ref. [27]. We define angle of emittance by $\alpha=\tan ^{-1}\left(p_{r} / p_{z}\right)$ as the angle between the electron ejection direction and the laser propagation direction along $z$ axis. We have investigated acceleration of 27th to 30th electrons of krypton atoms generated during ionization by a laser pulse in Fig. 9 which shows energy 

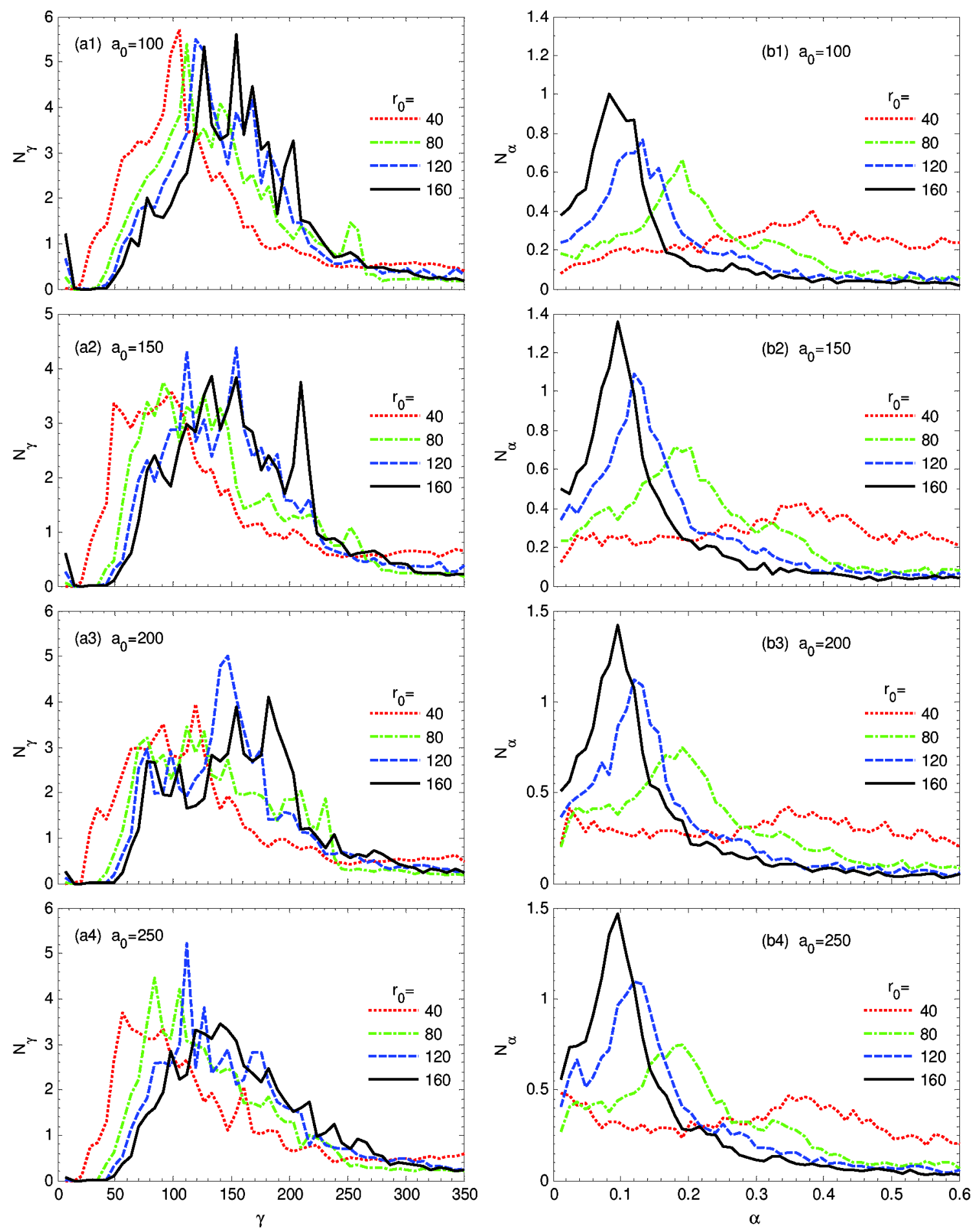

FIG. 9. Spectrum of (a1)-(a4) energy and (b1)-(b4) angle of emittance of the electrons generated during ionization of krypton by a laser pulse. The laser intensity parameters $a_{0}$ and laser spot size $r_{0}$ are specified in the figure itself. Here $N_{\gamma}$ and $N_{\alpha}$ are the number of electrons (relative scale) with energy lying within a range of relativistic factor and scattering lying within a range of angle of emittance, respectively.

spectrum and angle of emittance spectrum. The laser intensity parameters are (a1) $a_{0}=100,(\mathrm{a} 2) a_{0}=150$, (a3) $a_{0}=200$, and (a4) $a_{0}=250$ and different lines in the figures are for normalized laser spot size $r_{0}=40,80$, 120 , and 160 . There are several peaks corresponding to different laser intensities and laser spot sizes in the Figs. 9 (a1)-(a4). A sharper peak indicates lower spread in the energy and better beam quality. Some peaks are not very well defined and will not be described because beam quality is not good for these widely spread peaks. The 
peaks in energy spectrum at low laser powers are of major significance and particular interest. There is a well-defined peak in the energy spectrum at nearly $\gamma=100$ for $a_{0}=$ 100 and $r_{0}=40$ in Fig. 9 (a1) which is of major signifi- cance because it is at lowest laser power compared with other peaks in Fig. 9. There is a second well-defined peak in the energy spectrum at nearly $\gamma=150$ for $a_{0}=200$ and $r_{0}=120$ in Fig. 9 (a3) and a third peak at $\gamma=110$ for
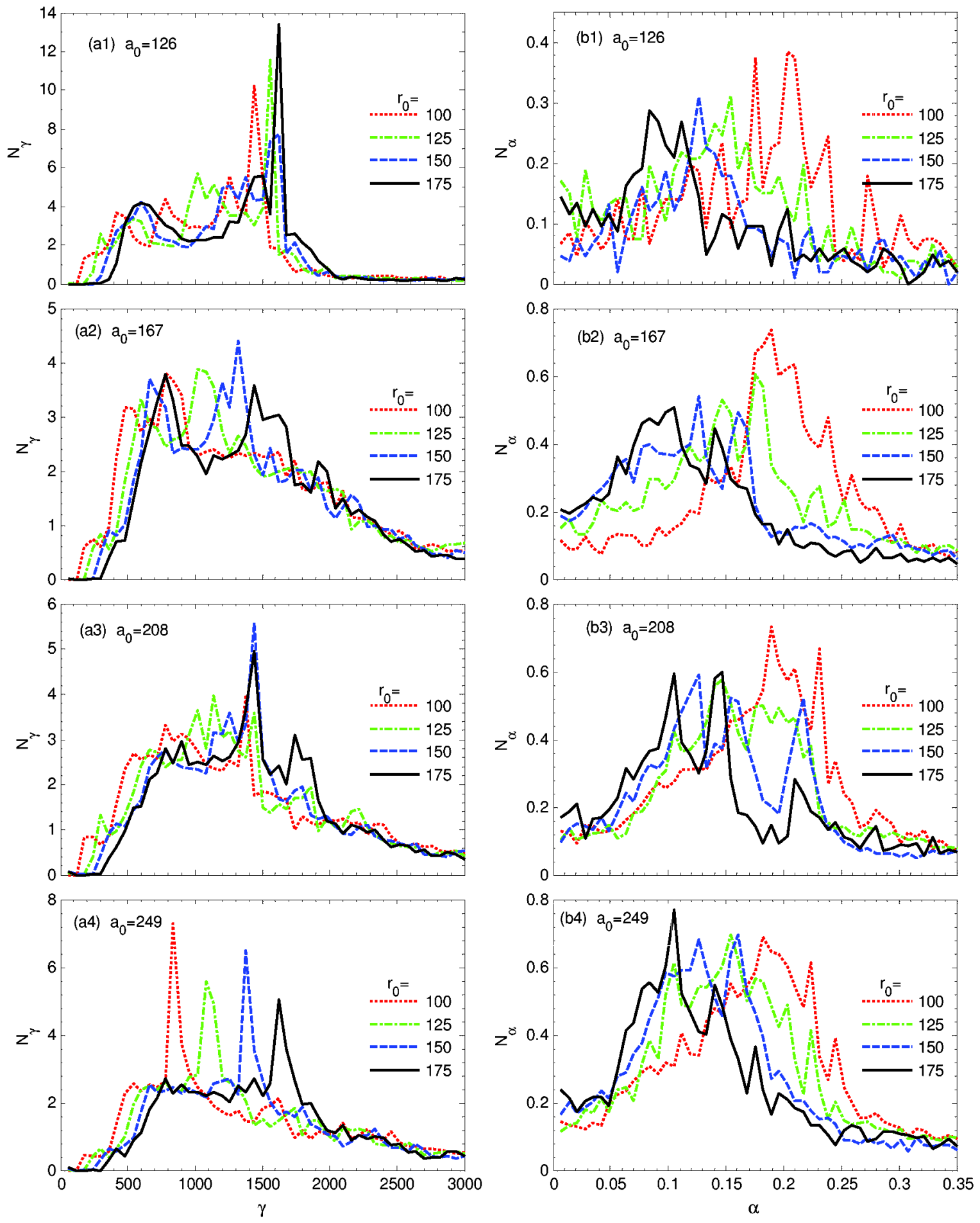

FIG. 10. Spectrum of (a1)-(a4) energy and (b1)-(b4) angle of emittance of the electrons generated during ionization of argon by a laser pulse. The laser intensity parameters $a_{0}$ and laser spot size $r_{0}$ are specified in the figure itself. Here $N_{\gamma}$ and $N_{\alpha}$ are the number of electrons (relative scale) with energy lying within a range of relativistic factor and scattering lying within a range of angle of emittance, respectively. 
$a_{0}=250$ and $r_{0}=120$ in Fig. 9 (a4). It can be seen from the Figs. 9 (b1)-(b4) that the angle of emittance is around $0.4^{\circ}$ corresponding to most of the peaks described in this paragraph. Overall, the value of angle of emittance is very small, which means we can obtain well collimated electron beams using radially polarized laser pulse.

Similar to krypton atoms, there is significant difference between the ionization energies of 16th $(918.03 \mathrm{eV})$ and 17 th $(4120.89 \mathrm{eV})$ electrons of the argon which can be used to obtain high energy electrons by removing 16 electrons from the outer shells of the argon by a low intensity prepulse and then using a main laser pulse to accelerate 17 th and 18th electrons from the atoms to high energies. Figures 10 (a1)-(a4) correspond to energy spectrum and Figs. 10 (b1)-(b4) correspond to the angle of emittance spectrum of the 17th and 18th electrons of argon atoms generated during ionization by a laser pulse. The laser intensity parameters for Figs. 10 (a1)-(a4) are $a_{0}=126$, $a_{0}=167, a_{0}=208$, and $a_{0}=249$, respectively, and different lines in the figures are for different values of laser spot size $r_{0}=100,125,150$, and 175 which are higher than those chosen for krypton. There are several peaks corresponding to different laser intensities and laser spot sizes in Figs. 10 (a1)-(a4) similar to those for krypton in Fig. 9. Some peaks are not very well defined and we will not describe them as before. There is a well-defined peak in the energy spectrum at nearly $\gamma=1400$ for $a_{0}=126$ and $r_{0}=100$ in Fig. 10 (a1). This peak is of major significance because it is at lowest laser power compared with other peaks shown in Fig. 10. There are other very well-defined peaks in Fig. 10 (a1) at higher values of normalized laser spot size which are not of interest due to requirement of higher laser powers with not a proportional increase in energy. There is a second very well-defined peak in the energy spectrum at nearly $\gamma=1400$ for $a_{0}=208$ and $r_{0}=150$ in Fig. 10 (a3). There are four very well-defined peaks in Fig. 10 (a4) for $a_{0}=249$ at $\gamma=850, \gamma=1100$, $\gamma=1375$, and $\gamma=1650$ for normalized laser spot sizes $r_{0}=100,125,150$, and 175 , respectively. It can be seen from Figs. 10 (b1)-(b4) that angle of emittance is around $0.2^{\circ}$ corresponding to most of the peaks in Fig. 10 .

We can conclude from the description of the previous two paragraphs related to krypton and argon gases that quasimonoenergetic collimated electron beams can be obtained using ionization of krypton and argon gases by radially polarized laser pulse. The peaks in the energy spectrum are sensitive to the laser intensity and laser size and a right of choice of parameters is needed to obtain good quality beams. The electron energy corresponding to the peaks in the spectrum increases with an increase in the normalized laser spot size and the angle of emittance decreases as the value of laser spot size increases for both krypton and argon gases. The findings in this article need to be tested experimentally to confirm choice of parameters to obtain quasimonoenergetic collimated elec- tron beams. The energy and collimation of the electrons is higher for argon than that for krypton due to lower atomic number and higher ionization potential for argon as compared to the krypton for inner shells which results in generation of more electrons close to the laser pulse peak for argon. Argon is therefore the preferred gas to obtain quasimonoenergetic, collimated high energy electron beams. We can obtain quasimonoenergetic collimated high energy electron beams using other gases as well; however, the energy may not be as high as for krypton and argon, and beam quality may not be that good because most of the other gases have lower ionization potential. The electrons from low atomic number gases will be generated at low laser intensities and will never interact with the peak of the high-intensity laser pulse; hence, the electrons would gain low energies as compared to krypton and argon. Other gases may be used to obtain low or medium energy quasimonoenergetic collimated electron for the applications which do not require energies in the range of $\mathrm{MeV}$ or $\mathrm{GeV}$.

\section{CONCLUSIONS}

The acceleration of electrons by a radially polarized intense laser pulse has been studied. The laser spot size, laser pulse duration, initial phase, and relative position of the electrons with respect to laser pulse affect electron acceleration. A right choice of laser spot size and injection is needed to achieve $\mathrm{GeV}$ electrons. The spatial profile of radial field intensity shows two lobes on both sides of $r=0$. The radial field intensity is highest for $r=r_{0} / \sqrt{2}$. The electrons follow a nearly straight line path which shows that acceleration is due to the longitudinal electric field of the laser. The longitudinal field intensity is maximum at $z=0$. Even though longitudinal electric field increases with decreasing laser spot size, the laser pulse gets defocused sooner for smaller values and does not experience high electric field for long reducing the energy. Electron energy peaks for the laser spot size $r_{0}=a_{0}$ because for this value the electron remains confined in the electric field of the laser for a long duration. Electron energy decreases for the electrons originating away from the axis of the laser pulse. Electron energy peaks for initial laser phase $\phi_{0}=\pi$ due to the accelerating phase of the laser. The electrons generated close to the peak of laser pulse gain higher energy than other electrons. The initial phase dependence is also shown by the electrons lying along the axis of the laser pulse. The energy and angle of emittance spectrum of the electrons generated during ionization of krypton and argon at low densities indicate that argon is more suitable to obtain high energy electron beams than krypton. Higher ionization potential of inner shells for the argon leads to generation of more electrons close to the laser peak which leads to higher energy. A right choice of laser parameters is needed to obtain high energy quasimonoenergetic collimated electron beams. 


\section{ACKNOWLEDGMENTS}

This work has been supported and acceleration simulation code used in this work has been developed by Singh Simutech Pvt. Ltd., Bharatpur, Rajasthan, India. The authors are thankful to Professor Peter A. Robinson, School of Physics, University of Sydney, New South Wales 2006, Australia, for fruitful discussions.

[1] P. B. Corkum and F. Krausz, Nature Phys. 3, 381 (2007).

[2] S.P.D. Mangles, C. D. Murphy, Z. Nujmudin, A. G. R. Thomas, J. L. Collier, A.E. Dangor, E. J. Divall, P. S. Foster, J. G. Gallacher, C. J. Hooker, D. A. Jaroszynski, A. J. Langley, W. B. Mori, P. A. Norreys, F. S. Tsung, R. Viskup, B.R. Walton, and K. Krushelnick, Nature (London) 431, 535 (2004).

[3] C. G. R. Geddes, Cs. Toth, J. Van Tilborg, E. Earey, C. B. Schroeder, D. Bruhwiler, C. Nieter, J. Cary, and W. P. Leemans, Nature (London) 431, 538 (2004).

[4] J. Faure, Y. Glinec, A. Pukhov, S. Kiselev, S. Gordienko, E. Lefebvre, J.P. Rousseau, F. Burgy, and V. Malka, Nature (London) 431, 541 (2004).

[5] B. Hafizi, P. Sprangle, and J. L. Hirshfield, Phys. Rev. E 50, 3077 (1994).

[6] A. Van Steenbergen, J. Gallardo, J. Sandweiss, and J. M. Fang, Phys. Rev. Lett. 77, 2690 (1996).

[7] W. D. Kimura, G. H. Kim, R. D. Romea, L. C. Steinhauer, I. V. Pogorelsky, K. P. Kusche, R. C. Fernow, X. Wang, and Y. Liu, Phys. Rev. Lett. 74, 546 (1995).

[8] E. Esarey, P. Sprangle, J. Krall, A. Ting, and G. Joice, Phys. Fluids B 5, 2690 (1993).

[9] T. Tajima and J. M. Dawson, Phys. Rev. Lett. 43, 267 (1979).

[10] Y. I. Salamin and C. H. Keitel, J. Phys. B 33, 5057 (2000).

[11] P. Sprangle, E. Esarey, and J. Krall, Phys. Plasmas 3, 2183 (1996).

[12] G. Malka, E. Lefebvre, and J. L. Miquel, Phys. Rev. Lett. 78, 3314 (1997).

[13] J. X. Wang, Y. K. Ho, Q. Kong, L. J. Zhu, L. Feng, S. Scheid, and H. Hora, Phys. Rev. E 58, 6575 (1998).

[14] K. P. Singh, D. N. Gupta and H. K. Malik, Phys. Scr. 77, 045401 (2008).

[15] K. P. Singh, D. N. Gupta, and V. Sajal, Laser Part. Beams 27, 635 (2009).

[16] L. W. Davis, Phys. Rev. A 19, 1177 (1979).
[17] J. P. Barton and D. R. Alexander, J. Appl. Phys. 66, 2800 (1989).

[18] Y. I. Salamin, G. R. Mocken, and C. H. Keitel, Phys. Rev. E 67, 016501 (2003).

[19] Y. I. Salamin, G. R. Mocken, and C. H. Keitel, Phys. Rev. ST Accel. Beams 5, 101301 (2002).

[20] L. Cicchitelli, H. Hora, and R. Postle, Phys. Rev. A 41, 3727 (1990).

[21] C. Varin, M. Piche, and M. A. Porras, J. Opt. Soc. Am. A 23, 2027 (2006).

[22] C. Varin and M. Piche, Phys. Rev. E 74, 045602 (2006).

[23] C. Varin, M. Piche, and M. A. Porras, Phys. Rev. E 71, 026603 (2005).

[24] Y. I. Salamin, Phys. Rev. A 73, 043402 (2006).

[25] D. N. Gupta, N. Kant, D. E. Kim, and H. Suk, Phys. Lett. A 368, 402 (2007).

[26] A. Karmakar and A. Pukhov, Laser Part. Beams 25, 371 (2007).

[27] Y. Kozawa, K. Yonezawa, and S. Sato, Appl. Phys. B 88, 43 (2007).

[28] Y. Kozawa and S. Sato, Opt. Lett. 30, 3063 (2005).

[29] J. Hamazaki, A. Kawamoto, R. Morita, and T. Omatsu, Opt. Express 16, 10762 (2008).

[30] T. Moser, J. Balmer, D. Delbeke, P. Muys, S. Verstuyft, and R. Baets, Appl. Opt. 45, 8517 (2006).

[31] P. L. Fortin, M. Piche, and C. Varin, J. Phys. B 43, 025401 (2010).

[32] S. Augst, D. Strickland, D. D. Meyerhofer, S. L. Chin, and J. H. Eberly, Phys. Rev. Lett. 63, 2212 (1989).

[33] P. B. Corkum, N.H. Burnett, and F. Brunel, Phys. Rev. Lett. 62, 1259 (1989).

[34] D. B. Milosevic, G. G. Paulus, D. Bauer, and W. Becker, J. Phys. B 39, R203 (2006).

[35] M. Ivanov, T. Seideman, P. Corkum, F. Ilkov, and P. Dietrich, Phys. Rev. A 54, 1541 (1996).

[36] C. I. Moore, A. Ting, T. Jones, E. Briscoe, B. Hafizi, R. F. Hubbard, and P. Sprangle, Phys. Plasmas 8, 2481 (2001).

[37] ELI-Extreme Light Infrastructure, European Project, http://www.eli-laser.eu.

[38] K. P. Singh, V. Sajal, and D. N. Gupta, Laser Part. Beams 26, 597 (2008).

[39] D. Ray, B. Ulrich, I. Bocharova, C. Maharjan, P. Ranitovic, B. Gramkow, M. Magrakvelidze, S. De, I. V. Litvinyuk, A. T. Le, T. Morishita, C. D. Lin, G. G. Paulus, and C. L. Cocke, Phys. Rev. Lett. 100, 143002 (2008). 